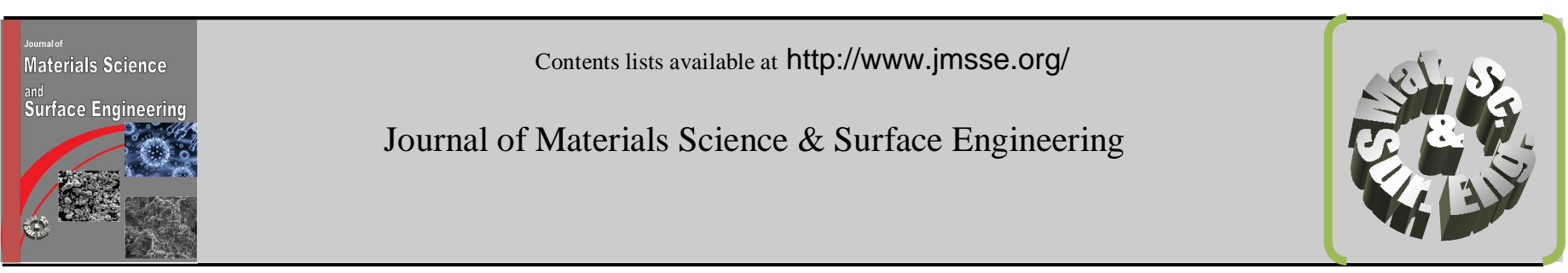

\title{
Surface Modification of Ag Nano Composites Polymer Membranes by Glow Discharge Plasma
}

\author{
N. K. Agrawal ${ }^{1}$, R. Agarwal $^{2}$, Y. K. Vijay ${ }^{3}$ and K. C. Swami ${ }^{1}$ \\ ${ }^{1}$ Department of Physics, Malaviya National Institute of Technology, Jaipur 302017, India. \\ ${ }^{2}$ Centre for Converging Technologies, University of Rajasthan, Jaipur - 302004, India. \\ ${ }^{3}$ Department of Physics, University of Rajasthan, Jaipur-302004, India.
}

Article history

Received: 30-Nov-2013

Revised: 29-Dec-2013

Available online: 06-Jan.- 2014

\section{Keywords:}

Ag Nano particles, Nano

composites, Ion Energy,

Plasma Etching Time.
Abstract

Nano particles of silver has been synthesized by Mangifera indica (mango) plant extract and characterized using UV-Vis spectrophotometer, FTIR (Fourier Transform Infrared Spectroscopy), XRD (X-ray Diffraction) and SEM (Scanning electron microscope). These nanoparticles were used as nano composites for polymer membranes. Using solution casting and spin coating method, Ag nano composite polymeric membranes in the range of 20-40 micron were prepared. Helium ion glow discharge plasma treatment was done for the membranes. Surface modifications for membranes were characterized before and after plasma to make comparative study by different technique such as optical microscopy, SEM- Scanning electron microscope. Results are discussed in this paper.

\section{Introduction}

Metallic nanoparticles synthesized traditionally by wet chemical synthesis where the chemicals used are often toxic and flammable ${ }^{1}$. Noble metal nanoparticles are widely used in many biological applications $^{2}$. So, there is a growing need to develop environmentally friendly processes for nanoparticle synthesis without using toxic chemicals. Biological methods of nanoparticle synthesis using micro-organisms, plants including algae, fungi, bryophyte, pteridophyta etc. have been suggested as possible ecofriendly alternatives to chemical methods ${ }^{3}$. Among the various inorganic metal nanoparticles, silver nanoparticles have received substantial attention for various reasons - like magnetic and optical property, electrical conductivity, catalysis, antimicrobial activities ${ }^{4-}$ 6. The use of silver metal ions for their sustained antifungal and antibacterial effects has been practiced ${ }^{7-10}$. In the present study Ag NPs were synthesized using Mangifera indica (mango) plant extract and characterized by UV-Vis spectrophotometer, FTIR, XRD and SEM.

Surface properties of polymers membranes not allow permanent bonding, coating, printing, etc., so it is very difficult to use on many polymers without surface pre-treatment in many applications ${ }^{11}$.Also after the surface treatment of polymers these have significant benefits in the field specific requirements of surface properties while retaining bulk mechanical properties unaltered $^{12}$. The complex nature of plasma due to presence of ions, neutrals and radiation in the discharge makes low-temperature plasmas widely used in a growing number of materials fabrication processes including the etching of complex patterns and surface modifications of polymeric membranes ${ }^{13-14}$.

Plasma surface treatment usually refers to a plasma reaction that either results in modification of the molecular structure of the surface, or atomic substitution. Plasma treatment is a useful tool in modification of surface properties ${ }^{15}$. The accelerated electrons from the plasma have sufficient energy to induce cleavage of the chemical bonds in the membrane structure and to form macromolecule radicals, which subsequently initiate graft copolymerization $^{16}$. The use of low pressure plasma enables the modification of polymer materials. Various plasma components such as electrons, ions, radical etc. are involved in this process. These components react with the exposed surfaces. Since some parts of the surface are exposed to energies higher than the characteristic bond energy of polymers, these parts undergo scission reactions and form new bonding configurations on the surface $^{17}$. Plasma treatment of polymer surface causes not only a modification during the plasma exposure, but also leaves active sites on the surfaces which are subjected to post-reaction ${ }^{18-19}$.

\section{Experimental}

The plant material were collected from university campus and washed with sterile distilled water. The plant extract was prepared by taking $25 \mathrm{~g}$ of thoroughly washed plant material in a $250-\mathrm{mL}$ Erlenmeyer flask with $100 \mathrm{~mL}$ of distilled water, and then boiling the mixture for $10 \mathrm{~min}$ in a water bath. The leaf broth was then cooled and filtered through Whatman No.1 filter paper (pore size $25 \mu \mathrm{m}$ ). $10 \mathrm{~mL}$ of the prepared plant extract was added to $90 \mathrm{ml}$ of $1 \mathrm{mM}$ silver nitrate solution and incubated in a rotary shaker for 2 $\mathrm{h}$. The color of the solution changed from light yellow to brown indicating the formation of silver nanoparticles.

The reduction of pure $\mathrm{Ag}+$ ions was monitored by measuring the $\mathrm{UV}-\mathrm{V}$ is spectrum of the reaction medium at 2 hours after diluting a small aliquot of the sample into distilled water. UV Vis spectral analysis was done by using UV-Vis spectrophotometer (Shimadzu). After the complete reduction of $\mathrm{Ag}+$ ions by the plant extract, it was analyzed by FTIR spectrophotometer (IR Affinity-1 Shimadzu) in the range of 4000- $400 \mathrm{~cm}-1$ for knowing the possible functional groups responsible for the formation of silver nanoparticles. The dried mixture of silver nanoparticles was analyzed by an X' Pert Pro X-ray diffractometer (PAN alytical BV, 
The Netherlands) operated at a voltage of $40 \mathrm{kV}$ and a current of $30 \mathrm{~mA}$ with $\mathrm{Cu} \mathrm{K} \alpha$ radiation in a $\theta-2 \theta$ configurations. Scanning Electron Microscopic (SEM) analysis was done using Hitachi S4500 SEM machine. Thin films of the sample were prepared on a carbon coated copper grid by just dropping a very small amount of the sample on the grid and then the film on the SEM grid was allowed to dry.

Polycarbonate granules were used to prepare flat sheet membranes by solution cast method. They were obtained as commercial grade from Loxim Polymers, Jaipur. Dichloromethane of extra pure grade was used as a solvent for preparing $10 \%$ polymer solution. Solution-casting method was used for preparation of polycarbonate membranes (both doped and pristine). Polycarbonate granules are weighed and dissolved in dichloromethane $\left(\mathrm{CH}_{2} \mathrm{Cl}_{2}\right)$ to prepare a $10 \%$ solution. Agitation of the solution is important, since the solvent penetration is very slow for high molecular weight polymers and a viscous coating is usually formed over each particle. The solution is stirred by magnetic stirrer to ensure the uniform dissolution and to enhance the rate of dissolution ${ }^{20-21}$. The process is carried out at room temperature for around 2-3 hours till a clear solution is formed. The solution was then put into flat-bottomed Petri-dishes floating on mercury to ensure a uniform structure of the membranes. The solvent was allowed to evaporate slowly over a period of $10-12 \mathrm{~h}$. The films so obtained were peeled off using forceps. For $3 \% \mathrm{Ag}$ nano composites membrane; Ag nanoparticles were dispersed in the solvent dichloromethane using ultra-sonicator. This dispersed solution was then added to the polycarbonate solution and stirred for around 30 minutes.

Plasma treatment consists of a source chamber with the complete power supply, connected to a vacuum system. The magnet is positioned to get a magnetic field inside the source chamber. The helium gas used for containing plasma is admitted into source chamber using a flow controller and applying DC power between two electrodes in magnetic field. The confined plasma in the chamber is used for surface modification. In this study we have used He plasma. The plasma is almost homogenous in a low-pressure glow discharge. The reaction chamber is evacuated and then refilled with low-pressure $\mathrm{He}$ gas to create glow discharge plasma. The gas is then energized by direct current. The energetic species in plasma include ions, radicals, electrons and meta-stable photons in short-wave UV range.

\section{Results and Discussion}

UV-Visible absorption spectrum shows characteristic surface plasmon resonance (SPR) peak of Ag NPs at $412 \mathrm{~nm}$ (Figure 1), indicating reduction of silver nitrate and formation of $\mathrm{Ag}$ nanoparticles. Absorption bands obtained using FTIR are classified as (A) stretching vibration of $\mathrm{Ag} \mathrm{NPs}$ at $763 \mathrm{~cm}-1$, (B) $\mathrm{C}=\mathrm{C}$ unsaturated at $1285 \mathrm{~cm}-1,(\mathrm{C}) \mathrm{C}=\mathrm{O}$ stretching vibration at 1712 $\mathrm{cm}-1$, (D) $\mathrm{C}-\mathrm{H}$ stretching vibration $3579 \mathrm{~cm}-1$ of aromatic compounds (Figure 2). Biosynthesized silver nanoparticles were further confirmed by the characteristic peaks observed in XRD profile and morphological and structural view under the scanning electron microscope. The XRD study indicates the formation of silver (Ag) nanoparticles which show four intense peaks (Figure 3) and SEM image of silver nanoparticles show average particle size of $40-60 \mathrm{~nm}$ (Figure 4). This indicates that the sample contained mixed phase, cubic $\left(44.5^{\circ}\right.$ and $\left.77^{\circ}\right)$ and hexagonal structures (peaks at $38^{\circ}$ and $64.1^{\circ} 2 \theta$ angle, after comparing form GCPDF data base) of silver nanoparticles. UV-Visible absorption spectrum shows characteristic surface plasmon resonance (SPR) peak of Ag NPs at $412 \mathrm{~nm}$ (Figure 1), indicating reduction of silver nitrate and formation of $\mathrm{Ag}$ nanoparticles. Absorption bands obtained using FTIR are classified as (A) stretching vibration of $\mathrm{Ag}$ NPs at 763 $\mathrm{cm}-1$, (B) $\mathrm{C}=\mathrm{C}$ unsaturated at $1285 \mathrm{~cm}-1$, (C) $\mathrm{C}=\mathrm{O}$ stretching vibration at $1712 \mathrm{~cm}-1$, (D) C-H stretching vibration $3579 \mathrm{~cm}-1$ of aromatic compounds (Figure 2). Biosynthesized silver nanoparticles were further confirmed by the characteristic peaks observed in XRD profile and morphological and structural view under the scanning electron microscope. The XRD study indicates the formation of silver (Ag) nanoparticles which show four intense peaks (Figure 3) and SEM image of silver nanoparticles show average particle size of 40-60 $\mathrm{nm}$ (Figure 4). This indicates that the sample contained mixed phase, cubic $\left(44.5^{\circ}\right.$ and $\left.77^{\circ}\right)$ and hexagonal structures (peaks at $38^{\circ}$ and $64.1^{\circ} 2 \theta$ angle, after comparing form GCPDF data base) of silver nanoparticles.

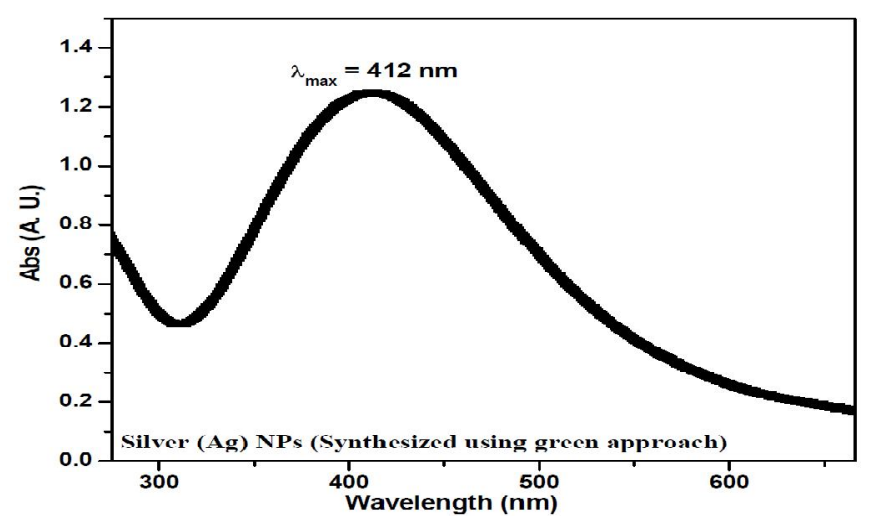

Figure 1: UV-Vis absorption spectrum of silver nanoparticles.

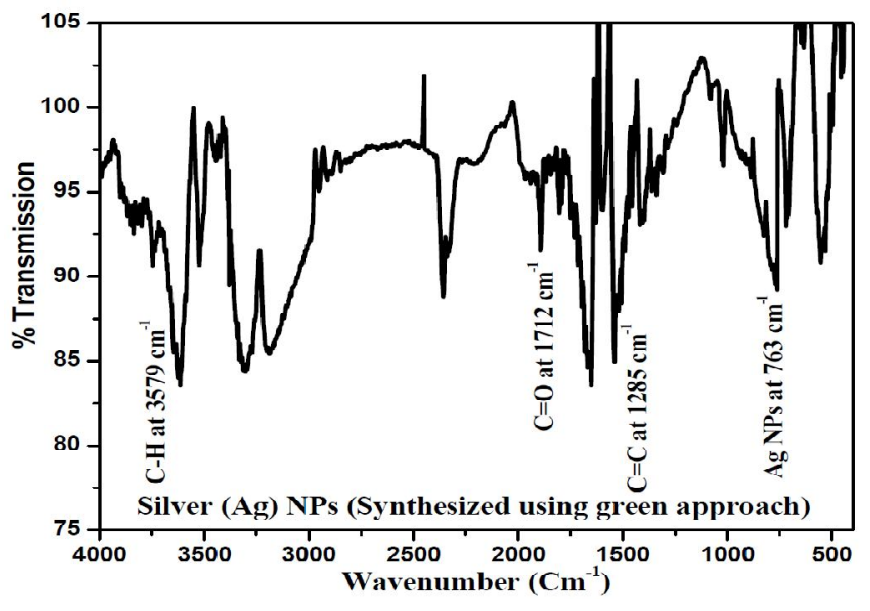

Figure 2: FTIR spectra of vacuum dried powder of silver nanoparticles

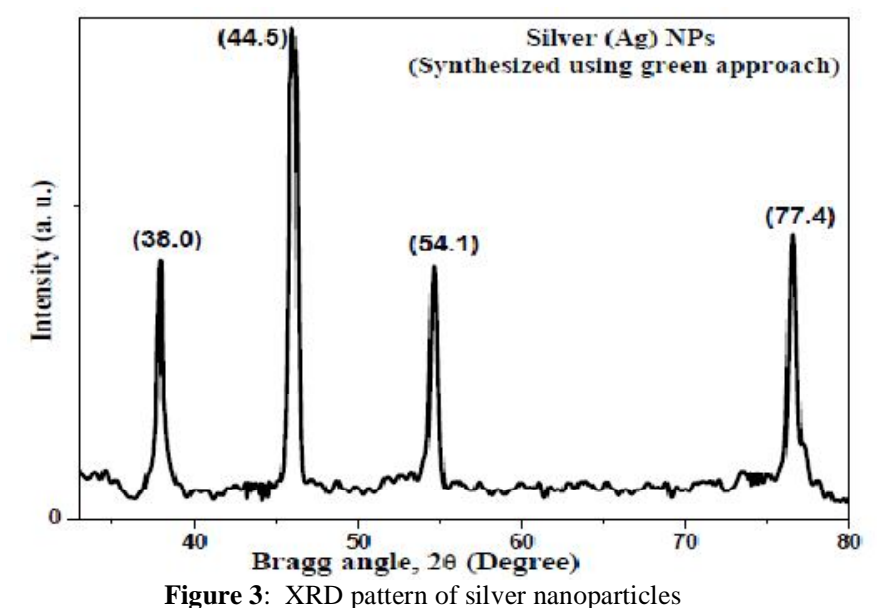




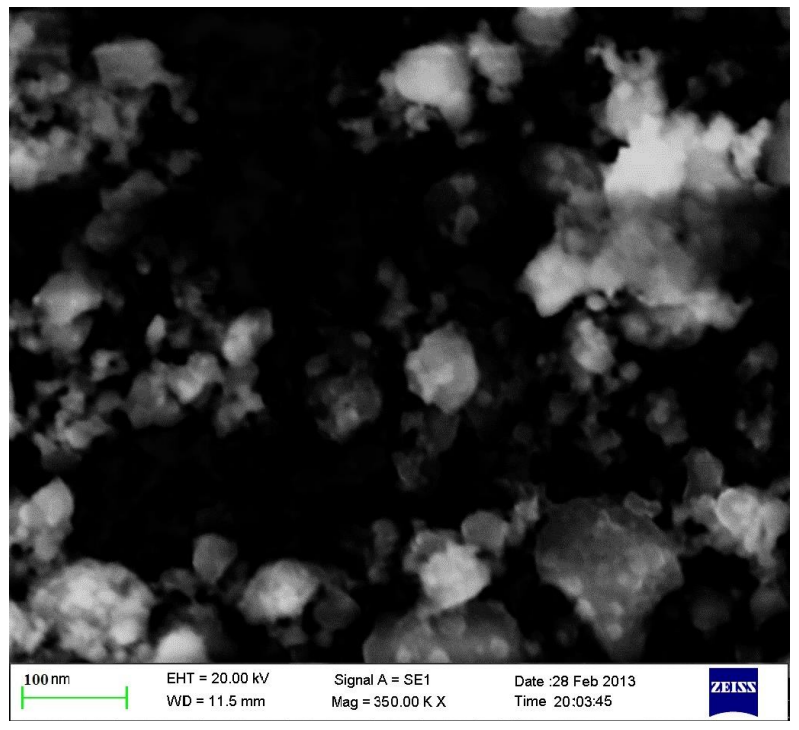

Figure 4: SEM image of silver nanoparticles

Surface modifications for membranes were characterized before
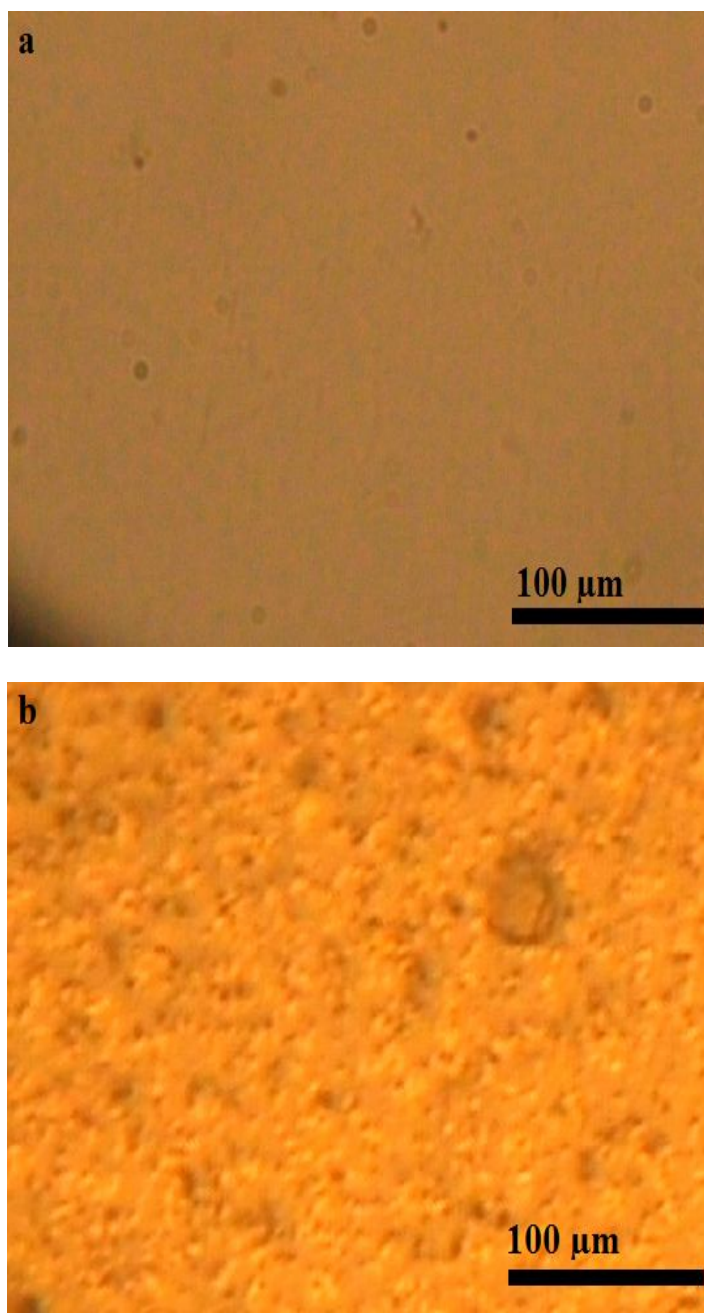

and after plasma to make comparative study by different technique such as optical microscopy, SEM- Scanning electron microscope. Optical microscope is the fundamental tool to study surface structures. It magnifies an image by sending a beam of light through the object. The eye-piece further magnifies the magnified image formed by the objective lens. An eyepiece does little to the primary image other than making it visible to the eye. LABOMED microscope is used for recording optical images. The micrographs are stored in computer through CCD camera which is attached to the computer with standard Pixel View software. Images shows that pristine membrane have very smooth surface but plasma treatment increase its roughness. Nano composite membrane is comparatively having high porosity but plasma treatment again increases its roughness (Fig. 5).

Surface morphologies of pristine polycarbonate membranes were investigated before and after plasma treatment and Ag Nano composite polycarbonate membran after plasma treatment using SEM. The SEM images of films are shown in Figure 6. We can easily say after looking the plasma treated membrane that it drastically change the surface property, this shows that plasma treatment techniques applied here have shown considerable improvement in surface morphology and flux. Plasma treatment has helped in increasing flux whereas doping has modified the surface properties (Figure 6).
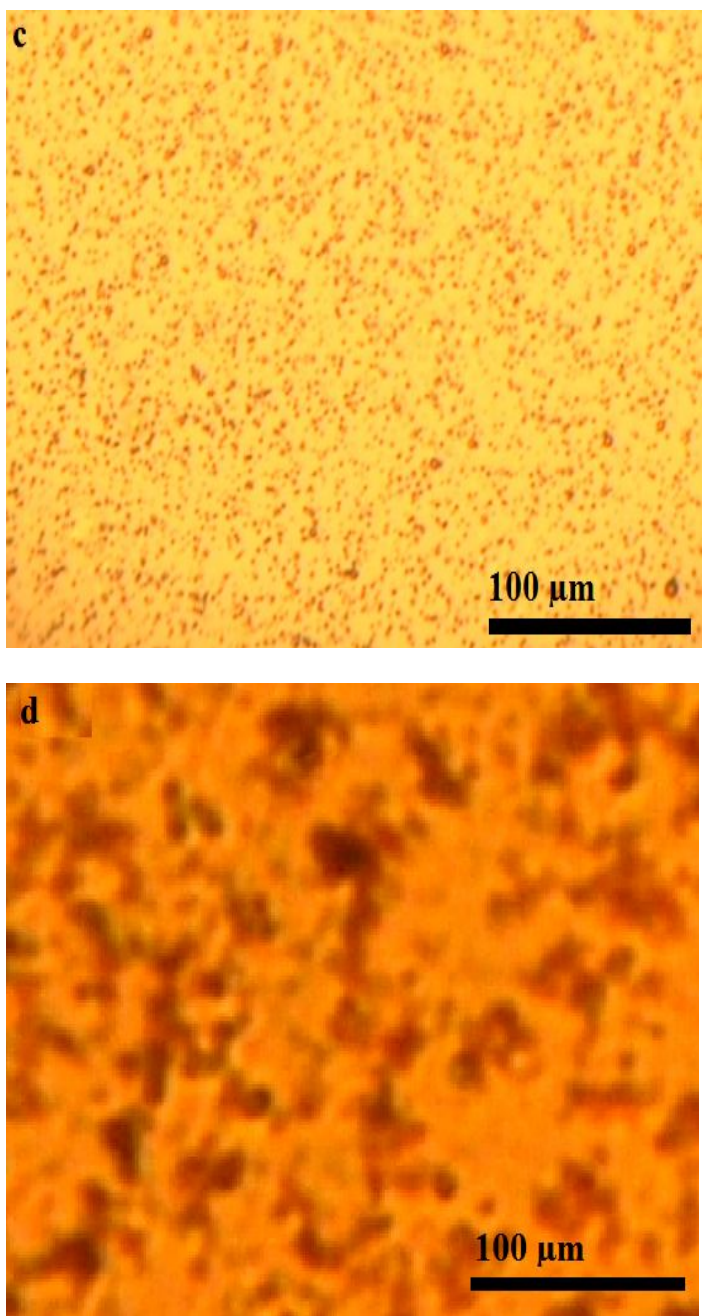

Figure 5: Optical microscope images of pristine and Ag nano composite both plasma treated and untreated (a) Pristine membrane, (b) $3 \% \mathrm{Ag}$ Nano composite membrane, (c) Plasma treatment pristine membrane, (d) Plasma Treated $3 \%$ Ag Nano Composite Membrane. 

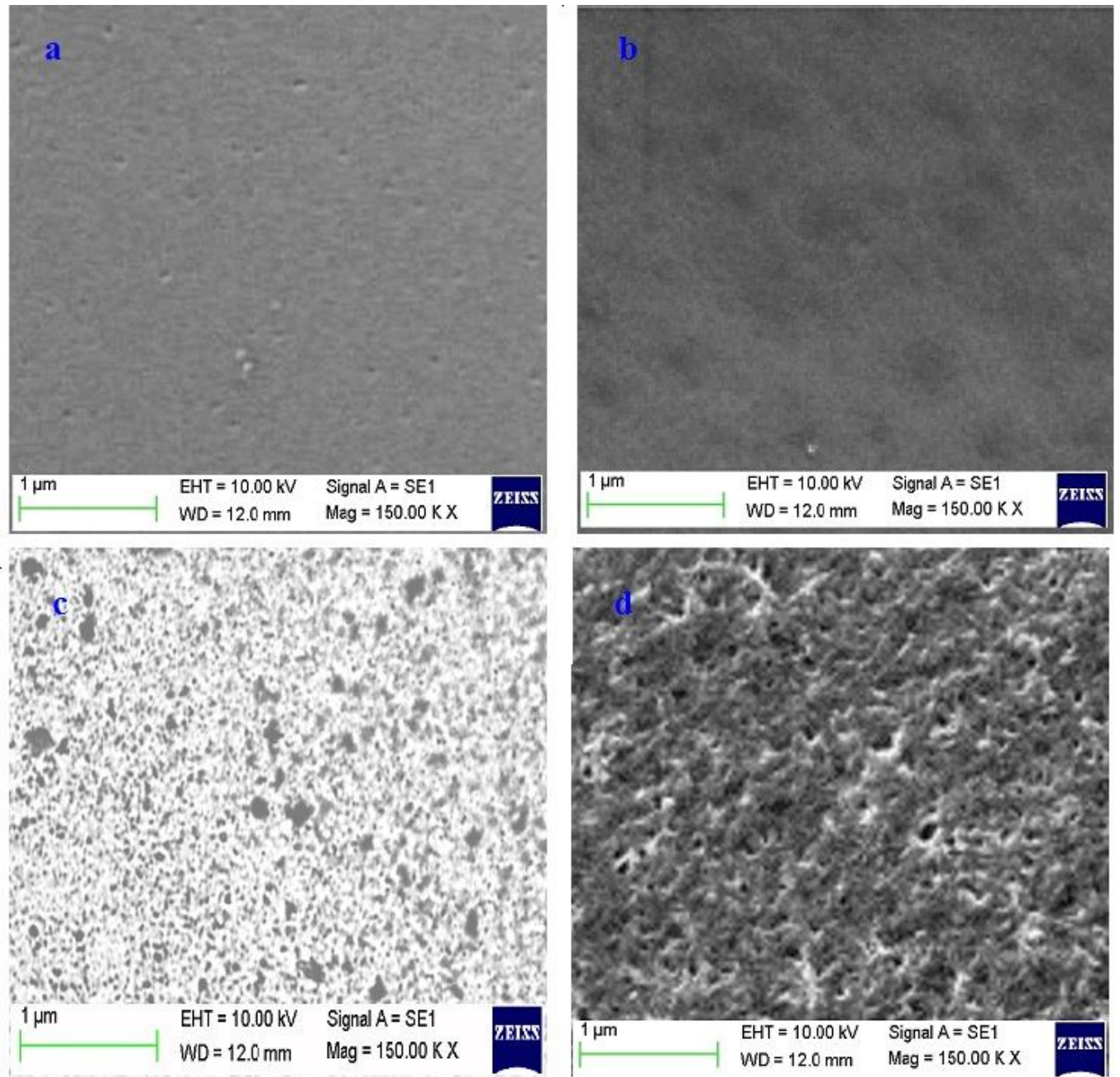

Figure 6: SEM images of pristine and Ag nano composite polymer membranes before and after plasma treatment (a) Pristine membrane, (b) 3\% Ag Nano composite membrane, (c) Plasma treatment pristine membrane, (d) Plasma Treated $3 \%$ Ag Nano Composite Membrane.

\section{Conclusions}

There was a visible color change from green to brownish as the plant extract was mixed in the aqueous solution of the silver ion complex which indicated formation of silver nanoparticles. Silver NPs formation was further confirmed by UV-Visible spectrophotometer by obtaining a spectrum in visible range of $300 \mathrm{~nm}$ to $800 \mathrm{~nm}$. A typical absorbance peak at $412 \mathrm{~nm}$ of silver nanoparticles was obtained due to the surface Plasmon vibrations of silver nanoparticles. Biosynthesized silver nanoparticles were further confirmed by the characteristic peaks observed in XRD profile and morphological and structural view under the scanning electron microscope. The XRD study indicates the formation of silver (Ag) nanoparticles which show four intense peaks and SEM image of silver nanoparticles show average particle size of 40-60 $\mathrm{nm}$. This indicates that the sample contained a mixed phase, cubic and hexagonal structures of silver nanoparticles. FTIR measurement carried out to identify the possible interaction between biomolecule and SNPs.

Pristine polycarbonate membranes and polycarbonate membrane doped with Ag nanoparticles were prepared by solution cast method. These membranes were subjected to surface modification techniques by He plasma treatment. Plasma treatment techniques applied here have shown considerable improvement in surface morphology and flux. Plasma treatment has helped in increasing flux whereas doping has modified the surface properties. As polycarbonate membrane is prepared without the help of any support, it can be concluded that polycarbonate has considerable strength as compared to other polymeric materials like polyamide which cannot be prepared without the help of support. An increase in surface roughness has been observed after plasma treatment by SEM and optical microscope images.

\section{Acknowledgement}

The authors wish to acknowledge to Department of Physics, University of Rajasthan for providing SEM measurement facility. Authors are also thankful to Jawaharlal Nehru Memorial Fund, New Delhi, India for providing Scholarship to Mr. Narendra Kumar Agrawal to carry out this work.

\section{References}

1. Y. Sanchuan, L. Xuesong, L. Jingqun, W. Dihua, L. Meihong and G. Congjie, Surface modification of thin-film composite polyamide reverse osmosis membranes with thermo-responsive polymer (TRP) for improved fouling resistance and cleaning efficiency; Separation and Purification Technology: 76, 2011, 283-291.

2. B. Bagra, P. Pimpliskar and N. K. Agrawal, Bio-Compatibility, Surface \& Chemical characterization of glow discharge plasma modified $\mathrm{ZnO}$ Nanocomposite Polycarbonate; proceedings of 58th DAE- Solid State Physics Symposium, AIP proceedings: India, 2013, Dec. 17-21.

3. P. Pimpliskar, B. Bagra, A. Sharma, S. Khandwal and N. K. Agrawal, An Innovative Approach for Efficient and Reusable Catalization for Synthesis of $\alpha, \beta$-Unsaturated Compounds Using Polymer - NPs Composites as Catalyst; proceedings of 58th DAE- 
Solid State Physics Symposium, AIP proceedings: India, 2013, Dec. 17-21.

4. L. Zou, I. Vidalis, D. Steele, A. Michelmore, S.P. Low and J.Q. Verberk, Surface hydrophilic modification of RO membranes by plasma polymerization for low organic fouling; Journal of Membrane Science: 369, 2011, 420-428.

5. N. K. Agrawal, N. A. Kumar, M. Singh, Y.K.Vijay and K.C.Swami, Plasma Surface Modification of Nano Composite Polymer Membranes: An Innovative Approach for Synthesis of Nano-Bio Materials; proceedings of 58th DAE- Solid State Physics Symposium, AIP proceedings: India, 2013, Dec. 17-21.

6. L. Meihong, Y. Sanchuan, Q. Ming, P. Qiaoming and G. Congjie, Impact of manufacture technique on seawater desalination performance of thin-film composite polyamide-urethane reverse osmosis membranes and their spiral-wound elements; Journal of Membrane Science: 348, 2008, 268-276.

7. H. Kanematsu, H. Ikegai and M. Yoshitake, Patents for Antibacterial Metallic Coating and Its Future Trend in Japan. Research Inventy; International Journal of Engineering and Science: 3(6), 2013, 47-55.

8. H. Kanematsu, Alloy Plating by Heating Stacked Single Layers and the Possibility of its Application in the Future; Products Finishing: 78(3), 2013, 1-14.

9. H. Kanematsu, H. Ikigai, and M. Yoshitake, Antibacterial Tin-Silver Plating by the Combination of Multistage Plating and Heat Treatment. The Journal of Applied Surface Finishing; 2008(3), 2008, 114-118.

10. P. Agarwal, R. Agarwal and N. K. Agrawal, Morphology and Crystallization of Size-Controlled Spherical Sliver Nanoparticles Synthesized Using Coriandrum sativum: A Greener Approach; proceedings of 58th DAE- Solid State Physics Symposium, AIP proceedings: India, 2013, Dec. 17-21.

11. W.M. King, P.A. Cantor, L.W. Schoellenback and C.R. Cannon, High-retention reverse-osmosis desalination membranes from cellulose acetate, Membranes from Cellulose Derivatives; Interscience Publisher: New York, 2001, 203-207.

12. N. K. Agrawal, R. Agarwal, Y. K. Vijay and K. C. Swami, Characterization of $\mathrm{N}_{2}$ Plasma Treated Nano Composites Polymer Membranes; Journal of Material Science Surface Engineering, 1 (1), 2013, 4-7.

13. P. Agarwal, A. Mehta, S. Kachhwaha and S. L.; Kothari, Cicer arietinum Leaf Extract Mediated Synthesis of Silver Nanoparticles and Screening of Their Antimicrobial Activity; Advanced Science, Engineering and Medicine: 5 (7), 2013, 709-714.

14. J.K. Beasley, The evaluation and selection of polymeric materials for reverse osmosis membranes, Desalination; Interscience Publisher: New York, 1997, 181-189.

15. R. Agarwal, N. K. Agrawal and Ramvir Singh, Cicer arietinum Leaf Extract Mediated Synthesis of Silver Nanoparticles and Screening of Its Antimicrobial Activity; Advanced Science, Engineering and Medicine: 6 (2), 2014, 203-207.

16. N. K. Agrawal, K. Awasthi, Y. K. Vijay and K. C. Swami, Synthesis and characterization of plasma treated $\mathrm{TiO}_{2}$ Nano composites polymer membranes; Journal of Advanced Electrochemistry: 2, 2013, 31-35.

17. K. Hyun and K. S. Soo, Plasma treatment of thin film composite reverse osmosis membrane; Journal of Membrane Science: 286, 2009, 193-201.

18. S Goel and S. K. Mathew, Automated Detection, Characterization, and Tracking of Sunspots from SoHO/MDI Continuum Images, Solar Phys: 32, 2013, 20-28.

19. N. K. Agrawal, M Singh, Y. K. Vijay and K. C. Swami, Synthesis and Characterization of Colloidal $\mathrm{TiO} 2$ Nanoparticles: Acquire Through Titanium Chloride Rich Solutions; Advanced Science, Engineering and Medicine: 7, 2014, 1-5.

20. N. K. Agrawal, R. Agarwal, Y. K. Vijay and K. C. Swami, Bacterial Cell Colonization on Nano Composites Polymer Membranes; Journal of Chemical and Biological Interfaces, (Accepted for publication).

21. N. K. Agrawal, R. Agarwal, Y. K. Vijay and K. C. Swami, Plasma etching technology for Surface and Chemical Modifications of Aluminium and PMMA Nanocomposites; Advanced Science, Engineering and Medicine; 6 (9), 2014, 520-528. 